\title{
Development of a novel resin with antimicrobial properties for dental application
}

\author{
Denise Tornavoi de CASTRO'1, Raphael Dias HOLTZ², Oswaldo Luiz ALVES ${ }^{2}$, Evandro WATANABE', Mariana Lima \\ da Costa VALENTE¹, Cláudia Helena Lovato da SILVA¹, Andréa Cândido dos REIS ${ }^{1}$
}

1- School of Dentistry of Ribeirão Preto, University of São Paulo, Ribeirão Preto, SP, Brazil.
2- State University of Campinas - UNICAMP, Campinas, SP, Brazil.

Corresponding address: Andréa Cândido dos Reis - Departamento de Materiais Dentários e Prótese - Faculdade de Odontologia de Ribeirão Preto Universidade de São Paulo - Av. do Café, s/nº - 14040-904 - Ribeirão Preto - São Paulo - Brazil - e-mail: andreare@forp.usp.br

Submitted: November 24, 2013 - Modification: April 22, 2014 - Accepted: May 1, 2014

\section{ABSTRACT}

$T^{\text {he }}$ he adhesion of biofilm on dental prostheses is a prerequisite for the occurrence of oral diseases. Objective: To assess the antimicrobial activity and the mechanical properties of an acrylic resin embedded with nanostructured silver vanadate $\left(\beta-\mathrm{AgVO}_{3}\right)$. Material and Methods: The minimum inhibitory concentration (MIC) of $\beta-\mathrm{AgVO}_{3}$ was studied in relation to the species Staphylococcus aureus ATCC 25923, Streptococcus mutans ATCC 25175, Pseudomonas aeruginosa ATCC 27853, and Candida albicans ATCC 10231. The halo zone of inhibition method was performed in triplicate to determine the inhibitory effect of the modified self-curing acrylic resin Dencor Lay - Clássico ${ }^{\circledR}$. The surface hardness and compressive strength were examined. The specimens were prepared according to the percentage of $\beta-\mathrm{AgVO}_{3}(0 \%$-control, $0.5 \%, 1 \%, 2.5 \%, 5 \%$, and $10 \%)$, with a sample size of $9 \times 2 \mathrm{~mm}$ for surface hardness and antimicrobial activity tests, and $8 \times 4 \mathrm{~mm}$ for the compression test. The values of the microbiologic analysis were compared and evaluated using the Kruskal-Wallis test $(\alpha=0.05)$; the mechanical analysis used the Shapiro-Wilk's tests, Levene's test, ANOVA (one-way), and Tukey's test $(\alpha=0.05)$. Results: The addition of $10 \% \beta-\mathrm{AgVO}_{3}$ promoted antimicrobial activity against all strains. The antimicrobial effect was observed at a minimum concentration of $1 \%$ for $P$. aeruginosa, $2.5 \%$ for $S$. aureus, $5 \%$ for C. albicans, and $10 \%$ for S. mutans. Surface hardness and compressive strength increased significantly with the addition of $0.5 \% \beta-\mathrm{AgVO}_{3}(\mathrm{p}<0.05)$. Higher rates of the nanomaterial did not alter the mechanical properties of the resin in comparison with the control group $(p>0.05)$. Conclusions: The incorporation of $\beta-\mathrm{AgVO}_{3}$ has the potential to promote antimicrobial activity in the acrylic resin. At reduced rates, it improves the mechanical properties, and, at higher rates, it does not promote changes in the control.

Keywords: Acrylic resins. Nanotechnology. Products with antimicrobial action.

\section{INTRODUCTION}

The installation of prosthetic devices within the oral cavity changes the oral conditions and promotes the deposit of biofilms on dental surfaces and on the prosthetic device ${ }^{25}$. The porous surface and irregularities of acrylic resins favor the accumulation of microorganisms, which are determining agents in the vast majority of oral problems, such as candidiasis, caries, gingivitis, and periodontitis ${ }^{28,29}$.

The solution to these problems includes the use of drugs and repairs or replacement of the prosthesis $4,5,7,8$. Prosthetic devices with antimicrobial properties could be ideal to improve the quality of life of patients and decrease discomfort and additional costs. With the addition of antimicrobial agents, several studies have focused on reducing or eliminating bacterial and fungal contamination ${ }^{8,9}$ without impairing the mechanical properties of dental materials.

Nanotechnology is currently the most promising field to generate new applications in healthcare, including dentistry ${ }^{6,13-20}$, with a special emphasis on silver nanoparticles $\left(\mathrm{Ag}^{\circ}\right)$. It is widely known for its antimicrobial properties and long application track record ${ }^{17}$. Therefore, compounds containing 
silver nanoparticles are effective because their broad-spectrum antimicrobial properties include the bacterium Streptococcus mutans, which is the main and most virulent cariogenic microorganism found in oral biofilm 2,6,18. A major problem with the use of AgNPs is the difficulty of homogeneously dispersing and incorporating the resin ${ }^{8}$; nanostructured silver vanadate is an alternative material for use in health care as it avoids the agglomeration of nanoparticles, providing high surface contact with the microorganisms and greater antibacterial activity $^{11}$.

This study is an unprecedented exploration of the effect of the addition of nanostructured silver vanadate on antimicrobial activity, surface hardness, and compressive strength of dental acrylic resin. The null hypothesis tested was that incorporating nanostructured silver vanadate would not affect the antimicrobial and mechanical properties of the acrylic resin. The antimicrobial behavior of the material was also determined and compared with the antimicrobial properties after the additive was incorporated $(0.5 \%, 1 \%, 2.5 \%$, $5 \%$, and $10 \%$ by mass).

\section{MATERIAL AND METHODS}

\section{Synthesis of nanostructured silver vanadate decorated with AgNPs}

The synthesis of the material was performed by a precipitation reaction between silver nitrate $\left(\mathrm{AgNO}_{3}\right.$, Merck $99.8 \%$ ) and ammonium metavanadate $\left(\mathrm{NH}_{4} \mathrm{VO}_{3} \text {, Merck 99\% }\right)^{12}$.

First, 0.9736 grams of $\mathrm{NH}_{4} \mathrm{VO}_{3}$ were dissolved in $200 \mathrm{~mL}$ of distilled water at $65^{\circ} \mathrm{C}$, under magnetic stirring for $10 \mathrm{~min}$. Next, 1.3569 grams of $\mathrm{AgNO}_{3}$ were dissolved in $200 \mathrm{~mL}$ of distilled water under the same conditions. The $\mathrm{AgNO}_{3}$ solution was added dropwise to the $\mathrm{NH}_{4} \mathrm{VO}_{3}$ and stirred for 30 min until it formed an ammonium vanadate solution. The precipitate obtained was filtered under vacuum, washed with distilled water and absolute ethanol, and dried in a vacuum line for 4 hours.

\section{Minimum Inhibitory Concentration}

The minimum inhibitory concentration (MIC) values of the nanostructured silver vanadate compared with the microorganisms Staphylococcus aureus ATCC 25923, Streptococcus mutans ATCC 25175, Pseudomonas aeruginosa ATCC27853, and Candida albicans ATCC 10231 (American Type Culture Collection, Manassas, VA, EUA) were determined based on successive dilutions outlined by the Clinical and Laboratory Standards Institute $(\mathrm{CLSI})^{7}$ in cell culture plates (TPP, Trasadingen, Unterklettgau, Switzerland) containing 96 wells. The plates contained the culture medium supplemented with increasing concentrations of the antimicrobial agent. Each well was inoculated with the microorganism under testing, and after 24 hours of incubation, the microbial growth was assessed by the turbidity of the culture.

\section{Experimental design}

The MIC of the nanostructured silver vanadate against microorganisms of interest has been determined, and it corresponds to the addition of nanomaterial to the resin structure. According to the literature ${ }^{11}$, the addition of $1 \%(\mathrm{w} / \mathrm{v})$ of silver vanadate to a commercial sample of water-based paint is sufficient to create antibacterial activity against Staphylococcus aureus. Thus, due to the unpublished nature of this study, to determine the effective concentration of vanadate silver incorporated in acrylic resin against proposed microorganisms, a greater number of concentrations were tested: (a) no antimicrobial agent, (b) $0.5 \%$ of $\beta-\mathrm{AgVO}_{3}$ (mass $/ \mathrm{mass}$ ), (c) $1 \%$ of $\beta-\mathrm{AgVO}_{3}$ (mass/ mass), (d) $2.5 \%$ of $\beta-\mathrm{AgVO}_{3}$ (mass $/$ mass), (e) $5 \%$ of $\beta-\mathrm{AgVO}_{3}$ (mass/mass), and (f) $10 \%$ of $\beta-\mathrm{AgVO}_{3}$ (mass/mass).

To test compressive strength, assuming a standard deviation of 7.95, an error of $5 \%$, and a power to $100 \% \mathrm{~N}=9$, it was necessary to perform a surface hardness test, assuming a standard deviation of 2.63, an error of $5 \%$, and power to $100 \% \mathrm{~N}=2$. Both analyses used $\mathrm{N}=10$ for each concentration of vanadate and control group.

For the antimicrobial analysis, 12 specimens were prepared for each concentration (vanadate and control group), which were distributed in groups of 3 specimens for each microorganism. The results of the analysis were replicated 3 times.

\section{Sample preparation}

The samples were prepared according to the specifications of the ISO (ISO 10477:1992/ Amd 1:1998) and followed the manufacturer's recommended proportions. The nanostructured silver vanadate samples were prepared by mixing $0.5 \%, 1 \%, 2.5 \%, 5 \%$, and $10 \%$ by mass of the antimicrobial agent with the polymer acrylic resin. After mixing the two powders, the monomer was added in the amount specified by the manufacturer, considering the sum of the two powders (polymer and vanadate).

Once it reached the plastic phase, the resin was adjusted to the muffle mold and pressed together using a hydraulic press (Midas Dental Products VH, Araraquara, SP, Brazil) gradually until reaching a pressure of $1000 \mathrm{Kgf}$, which was maintained during the curing time of the resin. The excess material was removed using a milling cutter (Maxi-cut, Maillefer AS, Ballaigues, Switzerland), followed by polishing with water sandpaper (Norton; SaintGobain Abrasivos Ltda, Vinhedo, SP, Brazil). 
Microbiologic analysis

The agar diffusion method was used to determine the inhibitory effect of the specimens on the proposed species.

The standardized microbial inocula were prepared in a saline solution $\left(\sim 10^{6} 10^{8} \mathrm{CFU} / \mathrm{mL}\right)$ from recent cultures at $37^{\circ} \mathrm{C}$ for $24 \mathrm{~h}$ using a spectrophotometer $(\lambda=625 \mathrm{~nm}$ and absorbance $=0.08$ to 0.100$)$. Next, $1 \mathrm{~mL}$ of these inocula was seeded onto the surface of Petri dishes (20x100 mm) containing $20 \mathrm{ml}$ of the culture medium, and the excess was removed with a pipette. The culture medium was Tryptic Soy Agar for S. mutans, Sabouraud Dextrose Agar for C. albicans, and Mueller Hinton Agar for S. aureus and $P$. aeruginosa.

The specimens were placed on the surface of the culture medium with the bacterial inoculum. Aliquots of $50 \mu \mathrm{L}$ of a chlorhexidine gluconate solution of $0.05 \%$, a positive control for antimicrobial activity, were applied to wells prepared in the center of the plates.

After the $2 \mathrm{~h}$ pre-incubation to disperse the products, the Petri dishes were incubated at $37^{\circ} \mathrm{C}$ for $24 \mathrm{~h}$ under aerobic conditions.

The zone of inhibition was determined by its diameter $(\mathrm{mm})$. The size of the zone of inhibition included the diameter of the specimen $(9 \mathrm{~mm})$.

\section{Compressive strength}

The specimens were submitted to a mechanical compressive strength test using the universal testing machine EMIC DL-2000 ${ }^{\circledR}$, with a load cell of $2000 \mathrm{kgf}$ and speed of $1 \mathrm{~mm} / \mathrm{min}$, according to ASTM D 695 and ISO 20795-1:2008. The Tesc ${ }^{\circledR}$ program, version 2.0, was used (EMIC, São José dos Pinhais, PR, Brazil), which stores the results in a graph database with numerical values in $\mathrm{MPa}$.

\section{Surface hardness}

The surface hardness test was carried with a Shimadzu microdurometer model HMV-2000 (Kyoto, Japan). Three random measurements were performed for each specimen with a Knoop diamond indenter under a load of $25 \mathrm{gf}$ for 5 seconds. The penetrations were measured by two markings on the vertices of the lozenge of a $40 x$ magnified image, which determined the length of the longest diagonal, and the Knoop hardness was automatically calculated by the software.

\section{Statistical analysis}

For the mechanical tests, once confirmed the normal distribution (Shapiro-Wilk's test) and homogeneous data (Levene's test), one-way ANOVA test and Tukey's test for comparison of means were performed.

The statistical analysis of microbiological assay was performed using the Kruskal-Wallis test.

For both analyses, a $p$ value of $<0.05$ was considered significant.

\section{RESULTS}

\section{Minimum inhibitory concentration}

Figure 1 shows the MIC results.

The MIC value compared with the strains of Staphylococcus aureus and Pseudomonas aeruginosa was $31.25 \mu \mathrm{g} / \mathrm{mL}$; for Candida albicans, it was $62.5 \mu \mathrm{g} / \mathrm{mL}$; and compared with Streptococcus mutans, it was $250 \mu \mathrm{g} / \mathrm{mL}$. These are important values as they demonstrate the antimicrobial effectiveness of silver vanadate against yeasts, gram-negative, and gram-positive bacteria responsible for oral problems commonly associated with dental prostheses.

\section{Antimicrobial activity}

Figure 2 shows the inhibition zone results. None of the negative control groups, with no addition of the antimicrobial agent, showed inhibitory activity.

Table 1 shows the average zones of inhibition diameter (in $\mathrm{mm}$ ) of the acrylic resin with different percentages of nanostructured silver vanadate, as well as the standard deviation and the differences between groups. For the acrylic resin specimens, there was an overall increase in the levels of inhibition with the increasing percentage of nanostructured silver vanadate.

The largest inhibition zone was observed with the addition of $10 \%$ in comparison with S. aureus $(15.6 \pm 0.6)$.

There was no statistically significant difference between the antimicrobial activity promoted by the addition of 5 to $10 \%$ compared with S. aureus,

\begin{tabular}{|c|c|c|c|c|}
\hline Code & Microorganisms & Source & Morphotintorial Characteristics & MIC $(\boldsymbol{\mu g} / \mathbf{m L})$ \\
\hline $\mathrm{Sa}$ & Staphylococcus aureus & ATCC 25923 & Gram-positive cocci & 31.25 \\
\hline $\mathrm{Sm}$ & Streptococcus mutans & ATCC 25175 & Gram-positive cocci & 250 \\
\hline $\mathrm{Pa}$ & Pseudomonas aeruginosa & ATCC 27853 & Gram-negative rods & 31.25 \\
\hline $\mathrm{Ca}$ & Candida albicans & ATCC 10231 & Gram-positive yeast & 62.5 \\
\hline
\end{tabular}

ATCC=American Type Culture Collection

Figure 1- Standard microorganisms used in the microbiological experiments and minimum inhibitory concentration (MIC) values for Ag vanadate 
$P$. aeruginosa, and C. albicans. Regarding $P$. aeruginosa, the percentage of $2.5 \%$ also showed microbial activity similar to 5 and $10 \%$ (Table 2).

To assist in understanding the differences between the groups, the descriptive statistical analysis of groups with greater than a 0.0 standard deviation is described.

\section{Compressive strength}

Table 3 shows the compressive strength values. Compared with the control group, a statistically significant difference $(p<0.05)$ was observed only for the group with $0.5 \%$ of the nanostructured silver vanadate, which showed the highest value $(119.34 \pm 5.72 \mathrm{MPa})$, and which was statistically similar to the group with $2.5 \%$. Compared to the other groups, there was no statistically significant difference between $0 \%, 1 \%$, and $5 \%$ and $10 \%$, $0 \%, 2.5 \%$, and $10 \%$.

\section{Surface hardiness}

Table 4 shows the surface hardness values. Compared with the control group, a statistically significant difference $(p<0.05)$ was observed for the group with $0.5 \%$ of the nanostructured silver vanadate, which showed the highest value (26.14 $5.13 \mathrm{HK})$. There was no statistically significant difference $(p>0.05)$ between the groups with $0 \%, 1 \%, 2.5 \%, 5 \%$, and $10 \%$.
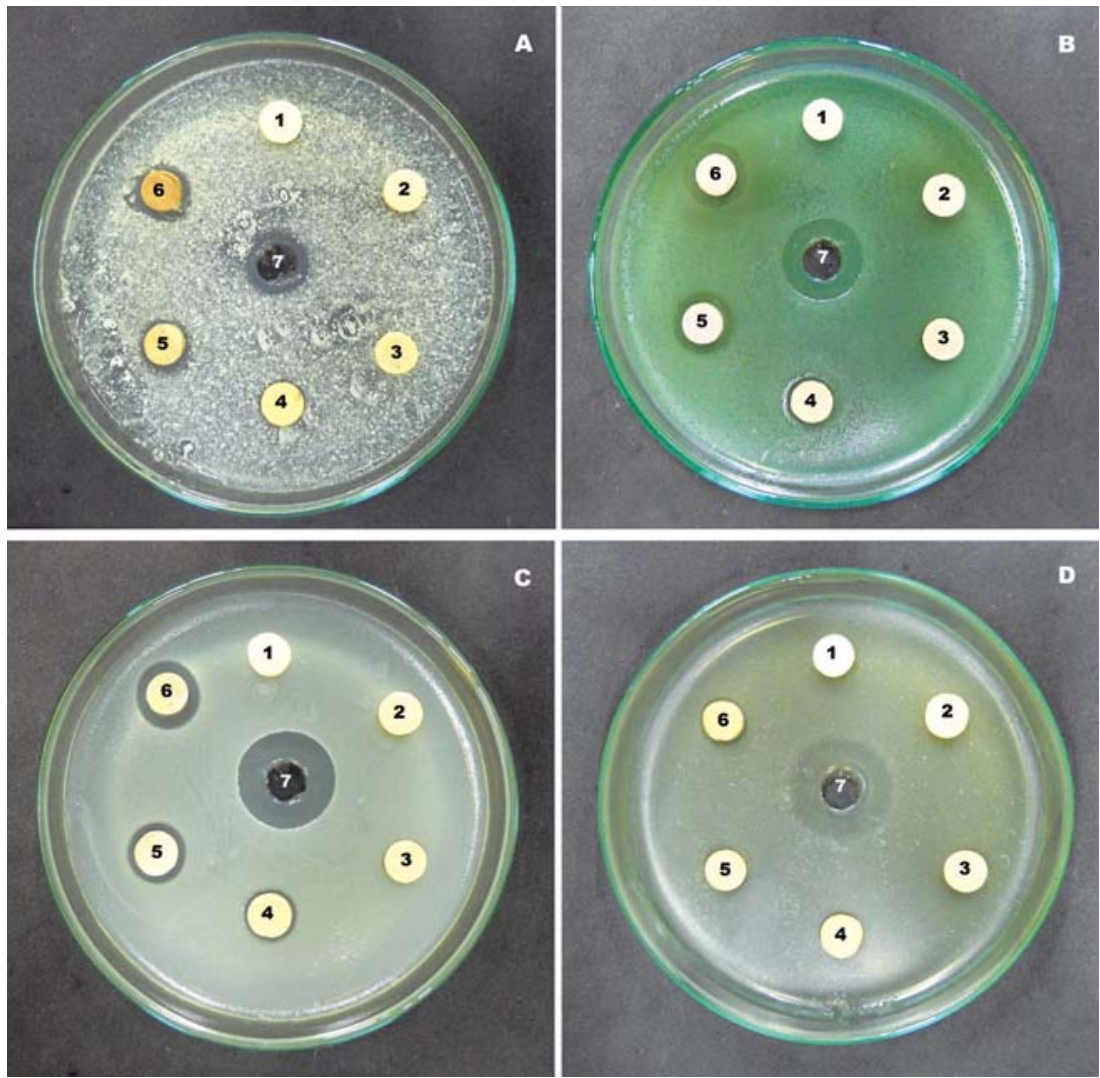

Figure 2- Zone of inhibition formed: A- Candida albicans (ATCC 10231); B- Pseudomonas aeruginosa (ATCC 27853); CStaphylococcus aureus (ATCC 25923); D- Streptococcus mutans (ATCC 25175). 1 - 0\%; 2 - 0.5\%; $3-1.0 \% ; 4-2.5 \%$; $5-5.0 \% ; 6-10 \%$. 7 - solution of chlorhexidine gluconate at $0.05 \%$

Table 1- Mean standard deviation (SD) of inhibition halos of self-cured acrylic resin incorporated with different percentages of nanostructured silver vanadate

\begin{tabular}{ccccccc}
\hline Microorganisms & $\mathbf{0 \%}$ & $\mathbf{0 . 5 \%}$ & $\mathbf{1 \%}$ & $\mathbf{2 . 5 \%}$ & $\mathbf{5 \%}$ & $\mathbf{1 0 \%}$ \\
\hline Candida albicans & $0.0(0.0)^{\mathrm{a}}$ & $0.0(0.0)^{\mathrm{a}}$ & $0.0(0.0)^{\mathrm{a}}$ & $0.0(0.0)^{\mathrm{a}}$ & $11.0(0.0)^{\mathrm{b}}$ & $12.3(0.6)^{\mathrm{b}}$ \\
Streptococcus mutans & $0.0(0.0)^{\mathrm{a}}$ & $0.0(0.0)^{\mathrm{a}}$ & $0.0(0.0)^{\mathrm{a}}$ & $0.0(0.0)^{\mathrm{a}}$ & $0.0(0.0)^{\mathrm{a}}$ & $12.0(0.0)^{\mathrm{b}}$ \\
Staphylococcus aureus & $0.0(0.0)^{\mathrm{a}}$ & $0.0(0.0)^{\mathrm{a}}$ & $0.0(0.0)^{\mathrm{a}}$ & $12.3(0.6)^{\mathrm{b}}$ & $14.6(0.6)^{\mathrm{c}}$ & $15.6(0.6)^{\mathrm{c}}$ \\
Pseudomonas aeruginosa & $0.0(0.0)^{\mathrm{a}}$ & $0.0(0.0)^{\mathrm{a}}$ & $10.6(9.1)^{\mathrm{b}}$ & $13.0(0.0)^{\mathrm{c}}$ & $14.6(0.6)^{\mathrm{c}}$ & $15.0(0.0)^{\mathrm{c}}$ \\
\hline
\end{tabular}

Different letters indicate significant differences between the groups for the same microorganism 
Table 2- Descriptive analysis

\begin{tabular}{cccccccc}
\hline & \% Nanomaterial & Minimum & Maximum & Mean & Median & SD & p value \\
\hline Candida albicans & $10 \%$ & 12.0 & 13.0 & 12.3 & 12.0 & 0.57 & 0.000 \\
Staphylococcus aureus & $2.5 \%$ & 12.0 & 13.0 & 12.3 & 12.0 & 0.57 & 0.000 \\
& $5 \%$ & 14.0 & 15.0 & 14.6 & 15.0 & 0.57 & \\
Pseudomonas aeruginosa & $10 \%$ & 15.0 & 16.0 & 15.6 & 16.0 & 0.57 & \\
& $1 \%$ & 1.0 & 19.0 & 10.66 & 12.0 & 9.07 & 0.000 \\
\hline
\end{tabular}

$\mathrm{SD}=$ standard deviation

Table 3- Mean standard deviation (SD) of compressive strength of self-cured acrylic resin incorporated with different percentages of nanostructured silver vanadate

\begin{tabular}{ccccccc}
\hline $\mathbf{0} \%$ & $\mathbf{0 . 5 \%}$ & $\mathbf{1 \%}$ & $\mathbf{2 . 5 \%}$ & $\mathbf{5 \%}$ & $\mathbf{1 0 \%}$ & $\mathrm{p}$ value \\
\hline 103.6 & 119.34 & 99.7 & 111.6 & 100.11 & 103.65 & 0.000 \\
$(8.9)^{\mathrm{ac}}$ & $(5.72)^{\mathrm{b}}$ & $(6.9)^{\mathrm{a}}$ & $(6.08)^{\mathrm{cb}}$ & $(8.44)^{\mathrm{a}}$ & $(9.44)^{\mathrm{ac}}$ & \\
\hline
\end{tabular}

Different letters indicate significant differences between the groups

Table 4- Mean standard deviation (SD) of surface hardness of self-cured acrylic resin incorporated with different percentages of nanostructured silver vanadate

\begin{tabular}{ccccccc}
\hline $\mathbf{0} \%$ & $\mathbf{0 . 5 \%}$ & $\mathbf{1 \%}$ & $\mathbf{2 . 5 \%}$ & $\mathbf{5 \%}$ & $\mathbf{1 0 \%}$ & $\mathbf{p}$ value \\
\hline 16.88 & 26.14 & 19.23 & 17.85 & 17.45 & 15.65 & 0.000 \\
$(2.0)^{\mathrm{a}}$ & $(5.13)^{\mathrm{b}}$ & $(1.41)^{\mathrm{a}}$ & $(2.11)^{\mathrm{a}}$ & $(1.71)^{\mathrm{a}}$ & $(1.61)^{\mathrm{a}}$ & \\
\hline
\end{tabular}

Different letters indicate significant differences between the groups

\section{DISCUSSION}

Biofilm formation on the surface of dental prostheses is a prerequisite for diverse microbial colonization and the occurrence of dental diseases ${ }^{28}$. It is related to factors such as the structure and composition of the surface material and the properties of microbial cells ${ }^{27}$. In addition, cleaning the prosthesis may be difficult to perform for geriatric patients who have cognitive impairment, reduced motor dexterity, and memory loss ${ }^{22}$. Thus, the need to develop a simple and effective system of care for the prosthesis exists, and an acrylic resin with antimicrobial properties could provide a significant advancement in the prevention of diseases and improvement of oral hygiene.

For this purpose, various substances have been incorporated into the acrylic resins for dentures and other dental materials ${ }^{27}$. These include antifungal agents ${ }^{6,8-23}$, antiseptics ${ }^{3}$, antimicrobial monomers ${ }^{24}$, and silver-based materials ${ }^{1}$. However, although some compounds showed good results, there is no reliable evidence in the literature to provide a true recommendation on the addition of these materials. An assessment of the actual effectiveness of the material, including the optimal therapeutic concentration, which does not deteriorate the mechanical properties of the substrate, produces no toxic effects on the oral cavity is required ${ }^{21-27}$.

Silver has a long history of application and is known to be superior to other metals and nonmetals in terms of antimicrobial properties. These properties are increased when prepared as nanoparticles. The combination of derivatives of ions and other silver compounds is highly toxic to microorganisms, but produces low toxicity for humans when in appropriate doses , $^{5-16}$.

Although studies show that dental materials incorporated with antimicrobial agents, when in contact with the oral cavity, lose their effectiveness quickly ${ }^{21-27}$, when silver-based materials are used, it is biologically safe and efficacious in the long term due to the slow release of ions ${ }^{30}$.

Even though the nanoparticles are important for diverse technical applications and provide several advantages when used alone, they have some common problems, such as the agglomeration of nanoparticles ${ }^{26}$. Thus, to expand the use of silver nanoparticles and improve some problems inherent to them, silver vanadate $\left(\mathrm{AgVO}_{3}\right)$ was developed, nanostructured, and decorated with AgNPs. 
This paper proposed the incorporation of this material into acrylic resin, promoting antimicrobial activity and increasing its mechanical properties. Once combined with $\beta-\mathrm{AgVO}_{3}$, the resin can potentially provide satisfactory results since this material presents high antibacterial activity in a broad spectrum of gram-positive and gramnegative bacteria ${ }^{11}$, and it functions as a carrier for silver nanoparticles (AgNPs). Contact with the microorganism changes its morphology and metabolism, leading to cell death ${ }^{20}$. It also reduces the loss of silver nanoparticles through leaching and has the form of a skein of yarn with nanometer dimensions. It can also adhere to the surface of the acrylic resin more easily than only AgNP12.

$\beta-\mathrm{AVO}_{3}$ also promotes a high dispersion of silver nanoparticles on nanowires of silver vanadates, which is important to maintain a large surface area with pathogenic microorganisms. This also has a greater effect and duration of action in terms of anti-microbial material characteristics ${ }^{11}$.

In this study, the control group samples (without the addition of nanostructured silver vanadate) showed no antimicrobial activity. As already demonstrated with other antimicrobial compounds, the effect was dose-dependent ${ }^{19-22}$. The addition of $10 \%$ of the nanomaterial was sufficient to produce antimicrobial activity against all strains tested, although at smaller percentages, the antimicrobial activity was observed in smaller proportions.

Some authors have suggested that the antimicrobial efficiency of polymers incorporated with silver-based materials is related to the release of silver ions or AgNPs; others report that the inhibitory effect is probably due to direct contact between the microorganisms and the material ${ }^{29}$. This may explain why the group with $10 \%$ of vanadate showed more sensitivity when compared to others, as a structure with more nanomaterial.

Marra, et al. ${ }^{19}$ (2012) incorporated an antimicrobial polymer acrylic resin and obtained good results using $10 \%$ and $25 \%$ against S. aureus and $S$. mutans, but there was no significant effect on C. albicans. In the present study, the antimicrobial effect was observed at a minimum concentration of $1 \%$ for $P$. aeruginosa, $2.5 \%$ for $S$. aureus, $5 \%$ for C. albicans, and $10 \%$ for S. mutans. The largest inhibition zone was observed with the addition of $10 \%$ against $S$. aureus $(15.6 \pm 0.6)$. Thus, the higher the vanadate percentage, the greater the antimicrobial activity. This shows that even relatively inert material, such as acrylic resin, can be modified to have useful antimicrobial properties.

In a study by Holtz, et al. ${ }^{11}$ (2012), adding $1 \%$ of the material to a water-based pigment was sufficient to promote a $4 \mathrm{~mm}$ zone of inhibition against $S$. aureus. With this same concentration in the acrylic resin, a small non-measurable inhibition against
S. aureus was observed around the specimen, and against $P$. aeruginosa, there was a greater halo of

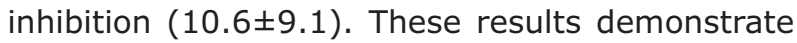
that although the acrylic resin matrix encapsulates the nanomaterial, it does not prevent it from acting on the microorganisms.

While promoting desirable effects in microbiological terms, the incorporation of antimicrobial agents in acrylic resin can promote undesirable changes in their mechanical and/or physical properties ${ }^{4-21}$. By incorporating methacrylate 2-tert-butylaminoethyl (TBAEMA) in acrylic resin, Paleari, et al. ${ }^{21}$ (2012) noted that the addition of $1 \%$ is sufficient to cause significant changes in flexural strength of the resin and justifies this change by incomplete polymerization of the same and permanence of residual monomer.

However, this study used a nanomaterial. The presence of unsaturated bonds on the nanomaterials' surface causes them to have high surface free energy, which also promotes strong bonding to other materials, hence promoting better physical and mechanical properties ${ }^{13}$. Thus, it was expected that in addition to promoting good antimicrobial activity, changing the composition of the resin would not reduce or improve the mechanical properties.

To confirm this hypothesis, the mechanical performance was evaluated in order to predict the durability of the material when exposed to forces in the oral cavity by compressive strength and surface hardness tests. These analyses are important because compressive forces are transmitted to the supporting tissues of the oral cavity. In the presence of parafunctional habits, this factor multiplies, with the frequent occurrence of fractures of prosthetic devices ${ }^{14}$, therefore laboratory compressive strength tests are helpful to observe in vitro the fractures that can occur in clinical practice ${ }^{15}$. While surface hardness represents the material's resistance to scratching, abrasion, or cutting, it is directly related to its wearing, which can result in more roughness and the adhesion of microorganisms $\mathrm{s}^{6,10-18}$.

The addition of $0.5 \%$ silver vanadate to the acrylic resin resulted in higher surface hardness and compressive strength values, indicating that a small percentage of nanometric particles can reinforce the material's matrix, which confirms the effectiveness of this material as a reinforcement structure, a widely required condition in dental materials. This percentage may be ideal to obtain this goal, though not to promote antimicrobial activity. Moreover, the addition of higher percentages promoted antimicrobial activity against microorganisms studied and has maintained the same values of surface hardness and compressive strength compared to the control group.

Significant results were found in the study. 
$\beta-\mathrm{AgVO}_{3}$ may be associated with acrylic resin as a potential antimicrobial agent, including $S$. mutans, the bacteria involved in the early stages of biofilm formation, and the production of extracellular polysaccharide, which facilitates connections with other microorganisms such as $C$. albicans (associated with denture stomatitis, or lesions commonly found in users of removable dentures) ${ }^{19}$. This association did not influence negatively the mechanical properties and is a low-cost method that does not depend on patient cooperation.

However, this study has some limitations. First, the literature is scarce on the subject, and no studies were found describing the incorporation of $\beta-\mathrm{AgVO}_{3}$ in polymers or other materials for dental applications or even its cytotoxicity in biological systems. Furthermore, only two mechanical properties were evaluated. One should evaluate the effect of incorporating other important mechanical properties, such as surface roughness and flexural strength, as well as the physical properties of the resin. Studies should include more complex biofilm models as well as cytotoxicity studies and longterm stability before these results can be applied in clinical research.

\section{CONCLUSIONS}

The incorporation of nanostructured silver vanadate in acrylic resin indicates possible antimicrobial activity directly related to the percentage of the nanomaterial applied to the microorganisms evaluated; $P$. aeruginosa and $S$. aureus are the most sensitive. Furthermore, the addition of $0.5 \%$ of the nanomaterial can promote improvement in the mechanical properties of hardness and resistance to compression.

\section{ACKNOWLEDGMENTS}

This study was conducted in partnership with: FAPESP (2012/09124-1 and 2012/09347-0); Solid State Chemistry Laboratory - UNICAMP; Oral Rehabilitation Research Laboratory - FORP/USP; Integrated Laboratory of Materials Biocompatibility Research - FORP/USP; Laboratory of Laser Welding and Corrosion Analysis - FORP/USP.

\section{REFERENCES}

1- Acosta-Torres LS, Mendieta I, Nuñez-Anita RE, CajeroJuárez M, Castaño VM. Cytocompatible antifungal acrylic resin containing silver nanoparticles for dentures. Int J Nanomedicine. 2012;7:4777-86.

2- Allaker RP. The use of nanoparticles to control oral biofilm formation. J Dent Res. 2010;89:1175-86.

3- Amin WM, Al-Ali MH, Salim NA, Al-Tarawneh SK. A new form of intraoral delivery of antifungal drugs for the treatment of dentureinduced oral candidosis. Eur J Dent. 2009;3(4):257-66.

4- Casemiro, LA, Gomes Martins CH, Pires-de-Souza FC, Panzeri $\mathrm{H}$. Antimicrobial and mechanical properties of acrylic resins with incorporated silver-zinc - part I. Gerodontology. 2008;25:187-94. 5- Cheng H, Li Y, Huo K, Gao B, Xiong W. Long-lasting in vivo and in vitro antibacterial ability of nanostructured titania coating incorporated with silver nanoparticles. J Biomed Mater Res A. 2014;102(10):3488-99.

6- Cheng L, Weir MD, Zhang K, Wu EJ, Xu SM, Zhou X, et al. Dental plaque microcosm biofilm behavior on calcium phosphate nanocomposite with quaternary ammonium. Dent Mater. 2012;28:853-62.

7- Clinical and Laboratory Standards Institute. Methods for dilution antimicrobial susceptibility tests for bacteria that grow aerobically. $6^{\text {th }}$ ed. Wayne: CLSI. 2003.

8- Fan C, Chu L, Rawls HR, Norling BK, Cardenas HL, Whang K. Development of an antimicrobial resin - a pilot study. Dent Mater. 2011;27:322-8.

9- Gjorgievska E, Apostolska S, Dimkov A, Nicholson JW, Kaftandzieva A. Incorporation of antimicrobial agents can be used to enhance the antibacterial effect of endodontic sealers. Dent Mater. 2013;29:e29-34.

10- Goiato MC, Santos DM, Baptista GT, Moreno A, Andreaotti AM, Dekon SF. Effect of thermal cycling and disinfection on microhardness of acrylic resin denture base. J Med Eng Technol. 2013;37:203-7.

11- Holtz RD, Lima BA, Souza Filho AG, Brocchi M, Alves OL. Nanostructured silver vanadate as a promising antibacterial additive to water-based paints. Nanomedicine. 2012;8:935-40. 12- Holtz RD, Souza Filho AG, Brocchi M, Martins D, Durán $\mathrm{N}$, Alves OL. Development of nanostructured silver vanadates decorated with silver nanoparticles as a novel antibacterial agent. Nanotechnology. 2010;21:185102.

13- Jandt KD, Sigusch BW. Future perspectives of resin-based dental materials. Dent Mater. 2009;25:1001-6.

14- Johansson A, Omar R, Carlsson GE. Bruxism and prosthetic treatment: a critical review. J Prosthodont Res. 2011;55:127-36. 15- Kamble VD, Parkhedkar RD. In vitro comparative evaluation of the effect of two different fiber reinforcements on the fracture toughness of provisional restorative resins. Indian J Dent Res. 2012;23:140-4.

16- Khurana C, Vala AK, Andhariya N, Pandey, OP, Chudasama B. Antibacterial activity of silver: the role of hydrodynamic particle size at nanoscale. J Biomed Mater Res A. 2014;102(10):3361-8. 17- Kumar V, Jolivalt C, Pulpytel J, Jafari R, Arefi-Khonsari, F. Development of silver nanoparticle loaded antibacterial polymer mesh using plasma polymerization process. J Biomed Mater Res A. 2013;101:1121-32.

18- Li F, Chen J, Chai Z, Zhang L, Xiao Y, Fang M, et al. Effects of a dental adhesive incorporating antibacterial monomer on the growth, adherence and membrane integrity of Streptococcus mutans. J Dent. 2009;37:289-96.

19- Marra J, Paleari AG, Rodriguez LS, Leite AR, Pero AC, Compagnoni MA. Effect of an acrylic resin combined with antimicrobial polymer on biofilm formation. J Appl Oral Sci. 2012;20:643-8.

20- Ozak ST, Ozkan P. Nanotechnology and dentistry. Eur J Dent. 2013;7:145-51. 
21- Paleari AG, Marra J, Pero AC, Rodriguez LS, Ruvolo-Filho A, Compagnoni MA. Effect of incorporation of 2-tert-butylaminoethy methacrylate on flexural strength of a denture base acrylic resin. J Appl Oral Sci. 2011;19:195-9.

22- Pesci-Bardon C, Fosse T, Serre D, Madinier I. In vitro antiseptic properties of an ammonium compound combined with denture base acrylic resin. Gerodontology. 2006;23:111-6.

23- Redding S, Bhatt B, Rawls HR, Siegel G, Scott K, Lopez-Ribot J. Inhibition of Candida albicans biofilm formation on denture material. Oral Surg Oral Med Oral Pathol Oral Radiol Endod. 2009;107:669-72.

24- Regis RR, Della Vecchia MP, Pizzolitto AC, Compagnoni MA Souza PP, Souza RF. Antimicrobial properties and cytotoxicity of an antimicrobial monomer for application in prosthodontics. J Prosthodont. 2012;21:283-90.

25- Rodriguez LS, Paleari AG, Giro G, Oliveira Junior NM, Pero AC, Compagnoni MA. Chemical characterization and flexural strength of a denture base acrylic resin with monomer 2-tert-butylaminoethyl methacrylate. J Prosthodont. 2013;22:292-7.
26- Shameli K, Ahmad MB, Zargar M, Yunus WM, Rustaiyan A Ibrahim NA. Synthesis of silver nanoparticles in montmorillonite and their antibacterial behavior. Int J Nanomedicine. 2011;6:58190.

27- Skupien JA, Valentini F, Boscato N, Pereira-Cenci T. Prevention and treatment of Candida colonization on denture liners: a systematic review. J Prosthet Dent. 2013;110:356-62.

28- Valentini F, Luz MS, Boscato N, Pereira-Cenci T. Biofilm formation on denture liners in a randomised controlled in situ trial. J Dent. 2013;41:420-7.

29- Wady AF, Machado AL, Zucolotto V, Zamperini CA, Berni E, Vergani CE. Evaluation of Candida albicans adhesion and biofilm formation on a denture base acrylic resin containing silver nanoparticles. J Appl Microbiol. 2012;112:1163-72.

30- Yu RY, Zhou YS, Feng HL, Liu XY. Silver-ion release and particle distribution of denture base resin containing nanometer-sized silver-supported antimicrobical agent. Zhonghua Kou Qiang Yi Xue Za Zhi. 2008;43(1):54-6. 